\title{
Discrete sources method for modeling the nonlocal optical response of a nonspherical particle dimer
}

\author{
Yuri Eremin ${ }^{\mathrm{a}}$, Adrian Doicu ${ }^{\mathrm{b}}$, Thomas Wriedt ${ }^{\mathrm{c}, *}$ \\ a Lomonosov Moscow State University, Lenin's Hills, Moscow 119991, Russia \\ ${ }^{\mathrm{b}}$ Remote Sensing Technology Institute German Aerospace Centre (DLR), Oberpfaffenhofen, Germany \\ ${ }^{\mathrm{c}}$ Leibniz-Institut für Werkstofforientierte Technologien - IWT, University of Bremen, Bremen, Germany
}

\section{A R T I C L E I N F O}

\section{Article history:}

Received 20 April 2018

Revised 25 May 2018

Accepted 25 May 2018

Available online 26 May 2018

\begin{abstract}
A B S T R A C T
A modified scheme of the discrete sources method for modeling the nonlocal optical response of a plasmonic dimer consisting of two identical axisymmetric metallic particles has been developed. The optical cross sections have been computed in the frequency domain for prolate spheroids with different aspect ratios and for different gap sizes. The results show that the generalized nonlocal optical response model leads to a blue shift, a plasmon mode damping, and a plasmon mode broadening of the optical cross sections as compared to the local-response approximation. These effects more pronounced when the aspect ratio of the spheroid increases and the gap size decreases. It has been confirmed that the additional boundary condition appears to be the main origin for the blue shift.
\end{abstract}

(c) 2018 Elsevier Ltd. All rights reserved.

\section{Introduction}

Interaction of light with plasmonic metallic nanostructures is of increasing interest in various research fields. The encountered effects are useful in different areas of science and technology, which include scanning near-field optical microscopy, metal enhanced fluorescent spectroscopy, biomedical sensors, optical data storage, solar cells and liquid crystal displays [1]. Plasmonics allows to manipulate light at the nanoscale and to obtain strong and very confined electromagnetic fields. This is achieved via a localized surface plasmon resonance, which is a well-known phenomenon that occurs in metal nanoparticles due to the collective oscillation of free electrons in nanosized structures when subjected to an incident electromagnetic field [2]. Interest in metallic structures with nanogap features has been propelled mostly by important applications in spectroscopy, and in particular, by the experimental reports of surfaceenhanced Raman scattering from single molecules [3]. The extreme level of sensitivity possible with such structures arises from the localized surface plasmon resonances that is associated with the nanogap, in which electric field intensity enhancements can reach large values.

The optical response of plasmonic nanostructures is generally well-described by classical electromagnetics based on the incorporation of local connection between displacement and electric field

\footnotetext{
* Corresponding author.

E-mail address: thw@iwt.uni-bremen.de (T. Wriedt).
}

by using frequency-dependent dielectric function. Such a classical approach produces predictive results for nanostructures until sizes down to $10 \mathrm{~nm}$. If the structure size is less than $10 \mathrm{~nm}$ or there is a sharp change of the surface topography the classical Maxwell's theory based on the local connection is no longer sufficient and a new approach is required going beyond classical electromagnetics to describe the occurring physical effects.

Plasmonic resonance modeling based on classical solution of Maxwell's equations predicts monotonically increasing electric field enhancements with decreasing the gap size, prompting the development of nanotechnology for producing plasmonic structures with nano sized gaps. The collective oscillation of conduction electrons subject to driving optical fields has been conceptually analyzed in the framework of the local response approximation (LRA), where the material response occurs only in the point of the perturbation. For pure dielectrics, this is a well-established and accurate approach, while it is traditionally being applied to metals too. Despite its simplifications, the LRA framework has fostered both predictions and experimental confirmations of novel plasmonic phenomena, such as the squeezing of light beyond the diffraction limit, the tunability of the optical properties of metallic structures with size and shape, and the large enhancement of the electric field in metal geometries with sharp surface topography corrugation, as well as in dimers with vanishing gaps [4]. In plasmonic nanogaps, three regimes for the gap size $d$ can be distinguished. 
1. For $d>2 \mathrm{~nm}$, the classical LRA gives basically the same results as a nonlocal response approximation (NLR) [5] (see Appendix for an overview of the nonlocal response theories).

2. For $0.5 \mathrm{~nm} \leq d \leq 2 \mathrm{~nm}$, strong nonlocal effects appear; both the local and nonlocal descriptions predict a similar qualitative behavior, but quantitative differences emerge.

3. For $d<0.5 \mathrm{~nm}$, pure quantum effects, such as the electron tunneling between the nanoparticles, are present [6,7].

Zuloaga et al. [8] used the time-dependent density-functiontheory (TDDFT) to perform fully quantum mechanical simulations of the linear response of plasmonic dimers. However, this approach is computationally unfeasible for large plasmonic structures (particle sizes exceeding $10 \mathrm{~nm}$ ) because the number of electrons to be accounted for is extremely large [9]. To address this situation, semi-classical approaches, in which quantum effects are incorporated into the Maxwell equations, have been proposed in [1015]. For large plasmonic structures with nanogaps, Esteban et al. [16] developed a quantum corrected model (QCM) to incorporate the electron tunneling effect into the local classical formalism. The quantum relationship between the oscillating field and current is reproduced by assigning a local effective conductivity to the gap. By analyzing the QCM results, it has been found that the "threshold tunneling-distance' is of about $4 \AA$. For larger gap sizes, the QCM results are identical to those of the LRA model as the tunneling effect is negligible [6]. David et al. [17] found that the extension of the hydrodynamic model to cope with inhomogeneous density profiles can provide a relatively fast and accurate way of describing the optical response of metal surfaces at subnanometer distances. Comparison results for gold sphere dimers are in a good agreement with well-established density-functional theory within the jellium model approach. An other approach called projected dipole model (PDM) can be applied to simulate dimers. PDM consists in placing an infinitely thin layer of dipoles on the corresponding surface. These dipoles are placed normal to the interface and their polarizabilities are defined by employing the time-dependent density-functional theory (TDDFT) within the jellium approximation and the following generalization for an arbitrary surface. As it was demonstrated by comparison with direct TDDFT computation for 2D dimers of arbitrary shapes PDM works well until vanishing gap size [18].

Along with electron tunneling, nonlocal screening [19] is another quantum effect that influences the plasmon resonances of a metallic dimer. Unlike the local classical model, in which the plasmon-induced charge densities are strictly confined to the surfaces, in the framework of a nonlocal hydrodynamic (NLHD) model, these charges are effectively pushed inside the material. This is a consequence of the choice of the boundary conditions on the free electron current at the metal-vacuum interface $[12,20]$. As a result, the plasmon resonances obtained with the NLHD model are always blue shifted with respect to the wavelengths predicted by the LRA model. The nonlocal screening effect also reduces the electric field enhancement in the gap of plasmonic dimers. The blue shift and the electric field enhancement predicted by the NLHD model correctly explain the measured effect of nonlocal screening for noble metals such as Au and Ag [7]. Mortensen et al. [11] proposed a generalized nonlocal response (GNOR) model in which an electron diffusion term is added to the NLHD model. This model predicts a progressive broadening of bonding modes in the subnanometer gap regime, which is in good agreement with the quantum TDDFT results [7]. To get a more physical insight into the role of the diffusion term incorporated in the GNOR description of the electron gas, the relaxation dynamics has to be considered. Under the influence of an external electric field, the free electrons in an initially charge-neutral metallic nanostructure are drawn away from their equilibrium positions. The electrons leaving regions near the sur- faces originate excess and deficit densities of charge. At the same time, of course, the whole bulk remains uncharged, since the positive ions remain fully screened by a corresponding density of negatively charged conduction electrons. As a consequence of entropy, the induced spatially inhomogeneous charge density will relax towards its equilibrium position. Classically, this process is described as diffusion, and in a drift-diffusion theory, it is captured by the diffusion constant. Since diffusion relaxes the induced charge, it also relaxes the polarization field, and consequently, it is a damping mechanism [21].

To provide reliable, accurate and controllable simulation results, a numerical electromagnetic model should account for all features of interaction in the plasmonic structures down to Angstrom dimension. An overview of the different numerical methods for analyzing nanoplasmonic structures can be found in [22-24]. The most popular approaches are (I) methods applicable directly to the Maxwell system (finite difference time domain [14,25] and finite elements method [26]), in particular in [14] the FDTD method was applied to analyze spherical dimers and arbitrary shaped cylindrical dimers, (II) semi-analytical volume based methods (discrete dipole approximation [24] and volume integral equations [23]), and (III) semi-analytical surface based methods (surface integral equations [23], T-matrix [27], multiple multipole method [28], and discrete sources method [29]). For plasmonic structures consisting of homogeneous plasmonic particles, surface based methods seem to be the most appropriate [23]. The reason is that the inclusion of the nonlocal response requires the solution of a vector Helmholtz equation with a very large wavenumber. Most direct methods when applied to the 3D case are restricted to a discretization step of about $0.2 \mathrm{~nm}$, which is not sufficient for modeling the nonlocal response. As a matter of fact, the discretization is determined by the Fermi wavelength inside the particle; for Ag, the Fermi wavelength $\lambda_{F}=0.52 \mathrm{~nm}$ requires a discretization step smaller than $0.1 \mathrm{~nm}$ to achieve a reasonable accuracy [30].

The discrete sources method (DSM) is a semi-analytical surface based method, in which the electromagnetic fields are approximated by linear combinations of discrete sources whose amplitudes are determined from the boundary conditions. As compared to other surface based approaches, the method (I) does not require any mesh generation or an integration procedure over the particle surface, (II) provides the near and far fields in clear analytical form, (III) solves simultaneously the scattering problem for all external excitations and polarizations, (IV) can handle particles with high refractive indices, and (V) allows the estimation of the computational errors via the residual of the surface fields [31]. These theoretical and numerical advantages recommend the discrete sources method for analyzing plasmonic structures in the presence of nonlocal response. It worth to mention that the DSM method can be applied considering "jellium edge" [17] as a set of discrete homogeneous shells, but in this case the DSM model simulation will be rather time consuming.

$>$ In this paper we analyze the plasmon resonances of a metallic dimer by means of a modified scheme of the discrete sources method which accounts on the nonlocal optical response. The paper is organized as follows. In Section 2 we summarize the generalized nonlocal optical response model, and formulate the transmission boundary value problem for a dimer consisting of two identical axisymmetric homogeneous metallic particles. Section 3 is devoted to the description of the modified scheme of the discrete sources method, while in Section 4, computersimulated results are reported and discussed.

\section{Generalized nonlocal optical response model}

We consider the scattering of an electromagnetic plane wave $\left(\mathbf{E}_{0}, \mathbf{H}_{0}\right)$ by a dimer consisting of two identical axisymmetric ho- 


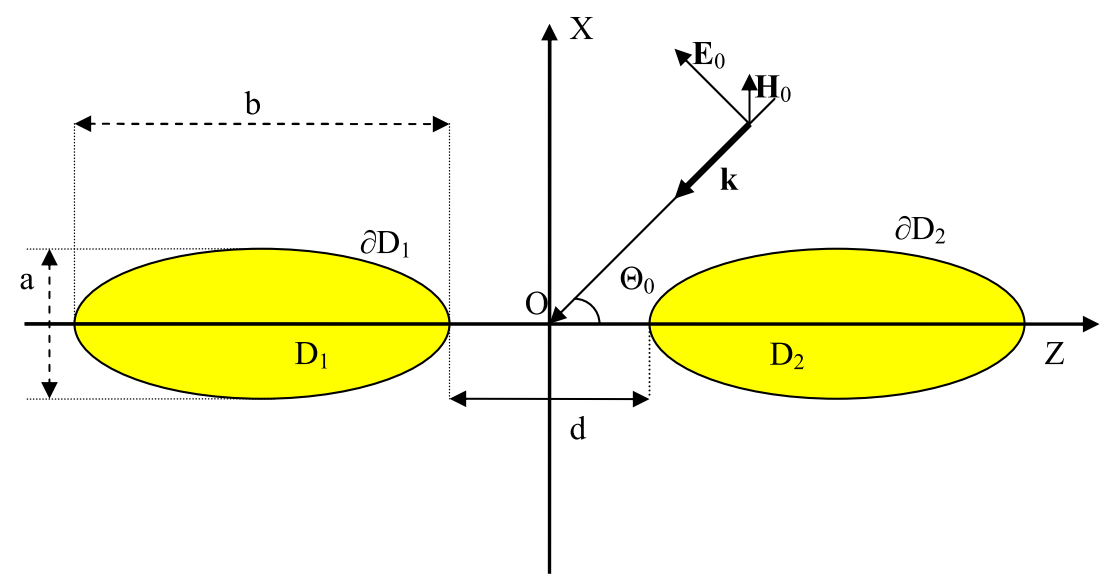

Fig. 1. Scattering geometry of a dimer consisting of two identical metallic prolate spheroids.

mogeneous metallic particles placed in an isotropic homogeneous medium as shown in Fig. 1. The particle $i$ occupies the domain $D_{i}$ with smooth boundary $\partial D_{i}$, where $i=1,2$. By assumption, the media are nonmagnetic and the particles have a common axis of symmetry $\mathrm{Oz}$. The incident electromagnetic plane wave propagates in the $O x z$ plane and along a direction which encloses the angle $\pi-\theta_{0}$ with the $O z$ axis.

An overview of the nonlocal response theories is given in the Appendix. In summary and referring to the GNOR model, the following conclusions are relevant for our analysis:

1. The local-response Ohm law $\mathbf{J}(\mathbf{r})=\sigma \mathbf{E}(\mathbf{r})$ is corrected for nonlocal dynamics as [12]

$\frac{\xi^{2}}{\varepsilon_{\mathrm{b}}} \nabla[\nabla \cdot \mathbf{J}(\mathbf{r})]+\mathbf{J}(\mathbf{r})=\sigma \mathbf{E}(\mathbf{r})$,

where for a time dependence $\exp (\mathrm{j} \omega t)$ of the fields,

$\xi^{2}=\varepsilon_{\mathrm{b}}\left[\frac{\beta^{2}}{\omega(\omega-\mathrm{j} \gamma)}+\mathrm{j} \frac{D}{\omega}\right]$

is the length scale of the GNOR model, $\varepsilon_{\mathrm{b}}$ the permittivity associated to bound charges, $\omega$ the frequency, $\beta^{2}=(3 / 5) v_{\mathrm{F}}^{2}, v_{\mathrm{F}}$ the Fermi velocity, $\gamma$ the Drude damping rate, $D$ the diffusion constant, and $\sigma$ the Drude conductivity.

2. The Maxwell equation for the magnetic field reads as

$\nabla \times \mathbf{H}(\mathbf{r})=\mathrm{j} \omega \varepsilon_{0}\left[\varepsilon+\xi^{2} \nabla(\nabla \cdot)\right] \mathbf{E}(\mathbf{r})$,

where

$\varepsilon=\varepsilon_{\mathrm{b}}-\frac{\omega_{\mathrm{p}}^{2}}{\omega^{2}-\mathrm{j} \gamma \omega}$

is the Drude permittivity, $\varepsilon_{0}$ the vacuum permittivity, and $\omega_{\mathrm{p}}$ the plasma frequency of the metal.

3. The field in the metal $\mathbf{E}$ sums the contributions of a divergencefree transverse field $\mathbf{E}_{\mathrm{T}}$ and a curl-free longitudinal field $\mathbf{E}_{\mathrm{L}}$ solving the vector Helmholtz equation with wavenumbers $k_{\mathrm{T}}^{2}=$ $k_{0}^{2} \varepsilon$ and $k_{\mathrm{L}}^{2}=\varepsilon / \xi^{2}$, respectively, where $k_{0}=\omega \sqrt{\varepsilon_{0} \mu_{0}}$ is the wavenumber in vacuum.

4. The amplitude of the longitudinal wave is computed from the additional boundary condition [12,35]

$\varepsilon_{1} \widehat{\mathbf{n}} \cdot \mathbf{E}_{1}(\mathbf{r})=\varepsilon_{\mathrm{b}} \widehat{\mathbf{n}} \cdot \mathbf{E}_{2}(\mathbf{r})$,

which is imposed at the interface between a dielectric medium 1 and a metallic medium 2. This boundary condition is used together with the continuity of the tangential components of the electric and magnetic fields at the interface.

In the framework of LRA, when nonlocal effects are neglected, the pertinent equations are obtained by setting $\xi=0$ in (1) and
(3), and by ignoring the longitudinal field (and so, the additional boundary condition (5)).

Considering the scattering problem illustrated in Fig. 1, we are faced with the solution of the following boundary value problem: Given the incident electromagnetic field $\left(\mathbf{E}_{0}, \mathbf{H}_{0}\right)$, compute the scattered field $\left(\mathbf{E}_{\mathrm{e}}, \mathbf{H}_{\mathrm{e}}\right)$ in $D_{\mathrm{e}}=\mathbb{R}^{3} \backslash \overline{D_{1} \cup D_{2}}$ and the internal fields $\left(\mathbf{E}_{i}, \mathbf{H}_{i}\right)$ in $D_{i}, i=1,2$, satisfying the Maxwell equations

$\nabla \times \mathbf{H}_{\mathrm{e}}=\mathrm{j} k_{0} \varepsilon_{\mathrm{e}} \mathbf{E}_{\mathrm{e}}, \quad \nabla \times \mathbf{E}_{\mathrm{e}}=-\mathrm{j} k_{0} \mathbf{H}_{\mathrm{e}}$, in $D_{\mathrm{e}}$

$\nabla \times \mathbf{H}_{i}=\mathrm{j} k_{0}\left[\varepsilon_{i}+\xi^{2} \nabla(\nabla \cdot)\right] \mathbf{E}_{i}, \quad \nabla \times \mathbf{E}_{i}=-\mathrm{j} k_{0} \mathbf{H}_{i}, \quad$ in $D_{i}, i=1,2$,

and the boundary conditions

$\widehat{\mathbf{n}}_{i} \times\left(\mathbf{E}_{i}-\mathbf{E}_{\mathrm{e}}\right)=\widehat{\mathbf{n}}_{i} \times \mathbf{E}_{0}$,

$\widehat{\mathbf{n}}_{i} \times\left(\mathbf{H}_{i}-\mathbf{H}_{\mathrm{e}}\right)=\widehat{\mathbf{n}}_{i} \times \mathbf{H}_{0}$,

$\varepsilon_{\mathrm{b}} \widehat{\mathbf{n}}_{i} \cdot \mathbf{E}_{i}=\varepsilon_{\mathrm{e}} \widehat{\mathbf{n}}_{i} \cdot\left(\mathbf{E}_{\mathrm{e}}+\mathbf{E}_{0}\right)$,

on $\partial D_{i}, i=1,2$. In addition, the scattered field must satisfy the Silver-Müller radiation condition. Here, $\varepsilon_{\mathrm{e}}$ is the permittivity of the background dielectric medium, and in order to further simplify the writing, we set

$\varepsilon_{1}=\varepsilon_{2}=\varepsilon$,

where $\varepsilon$ is given by (4). Note that the field inside particle $i$ is decomposed as

$\mathbf{E}_{i}=\mathbf{E}_{\mathrm{T} i}+\mathbf{E}_{\mathrm{L} i}$,

where $\mathbf{E}_{\mathrm{T} i}$ and $\mathbf{E}_{\mathrm{L} i}$ are respectively, the transverse and longitudinal fields satisfying

$\nabla \cdot \mathbf{E}_{\mathrm{Ti}}(\mathbf{r}, \omega)=0$ and $\nabla \times \mathbf{E}_{\mathrm{Li}}(\mathbf{r}, \omega)=0$.

\section{Discrete sources method}

In the framework of the discrete sources method, the electromagnetic fields are constructed as finite linear combinations of the fields produced by dipoles and multipoles distributed inside the particle. Thus, the solution satisfies Maxwell's equations and the radiation conditions analytically. The unknown amplitudes of the discrete sources are determined from the boundary conditions enforced at the particle surface. In this section we describe a modified scheme of the discrete sources method for modeling the nonlocal optical response. 
For the incident electromagnetic plane wave

$\mathbf{E}_{0}(\mathbf{r})=\widehat{\mathbf{e}}_{0} \mathrm{e}^{-\mathrm{j} \mathbf{k}_{\mathrm{e}} \cdot \mathbf{r}}$

propagating along the direction $\left(\pi-\theta_{0}, \pi\right)$ and being characterized by the wave vector $\mathbf{k}_{\mathrm{e}}=k_{\mathrm{e}} \widehat{\mathbf{k}}_{\mathrm{e}}, k_{\mathrm{e}}=k_{0} \sqrt{\varepsilon_{\mathrm{e}}}$ and polarization unit vector $\widehat{\mathbf{e}}_{0}$, the corresponding P- and S-polarized incident fields are given respectively, by

$\mathbf{E}_{0}^{\mathrm{P}}(\mathbf{r})=\left(\cos \theta_{0} \widehat{\mathbf{e}}_{\mathrm{x}}+\sin \theta_{0} \widehat{\mathbf{e}}_{\mathrm{z}}\right) \chi(\chi, z)$,

$\mathbf{H}_{0}^{\mathrm{P}}(\mathbf{r})=-\sqrt{\varepsilon_{\mathrm{e}}} \cos \theta_{0} \widehat{\mathbf{e}}_{\mathrm{y}} \chi(\chi, z)$,

and

$\mathbf{E}_{0}^{\mathrm{S}}(\mathbf{r})=\sqrt{\varepsilon_{\mathrm{e}}} \cos \theta_{0} \widehat{\mathbf{e}}_{\mathrm{y}} \chi(\chi, z)$,

$\mathbf{H}_{0}^{\mathrm{S}}(\mathbf{r})=\left(\cos \theta_{0} \widehat{\mathbf{e}}_{\mathrm{x}}+\sin \theta_{0} \widehat{\mathbf{e}}_{\mathrm{z}}\right) \chi(\chi, z)$,

where $\left(\widehat{\mathbf{e}}_{\mathrm{x}}, \widehat{\mathbf{e}}_{\mathrm{y}}, \widehat{\mathbf{e}}_{\mathrm{z}}\right)$ are the Cartesian unit vectors and

$\chi(x, z)=\mathrm{e}^{-\mathrm{j} k_{\mathrm{e}}\left(x \sin \theta_{0}-z \cos \theta_{0}\right)}$.

For an axisymmetric dimer, the P- and S-polarized excitations are treated separately. To account for axial symmetry, we expand each polarized plane wave into a Fourier series in the azimuthal angle $\varphi$ by using the basic result

$\mathrm{e}^{ \pm \mathrm{j} x \cos \varphi}=\sum_{m=0}^{\infty}\left(2-\delta_{m 0}\right)( \pm \mathrm{j})^{m} J_{m}(x) \cos m \varphi$,

where $J_{m}$ are the cylindrical Bessel functions, and $\delta_{m 0}$ the Kronecker delta symbol. The scattering problem then decouples over the azimuthal modes $m$, and a separate solution for each $m$ is obtained.

The discrete sources are placed on the axis $\mathrm{Oz}$ (the axis of symmetry of the dimer), and in this regard, we denote by $\left\{z_{n}^{\mathrm{e}}\right\}_{n=1}^{N_{\mathrm{e}}^{m}}$ the positions of discrete sources for the scattered field representation, by $\left\{z_{n}^{\mathrm{Ti}}\right\}_{n=1}^{N_{\mathrm{Ti}}^{m}}$ the positions of discrete sources for representing the transverse field inside particle $i$, and finally, by $\left\{z_{n}^{\mathrm{L} i}\right\}_{n=1}^{N_{\mathrm{L} i}^{m}}$ the positions of discrete sources for representing the longitudinal field inside particle $i$. Note that the numbers of discrete sources $N_{\mathrm{e}}^{m}, N_{\mathrm{T} i}^{m}$ and $N_{\mathrm{L} i}^{m}$ may depend on the azimuthal mode $m$.

For transverse field approximation, the discrete sources are constructed from the vector potentials

$$
\begin{aligned}
\mathbf{A}_{m n}^{1 \mathrm{e}, i}(\mathbf{r}) & =Y_{m}^{\mathrm{e}, i}\left(\varrho, z_{n}^{\mathrm{e}, \mathrm{Ti}}\right) \cos [(m+1) \varphi] \widehat{\mathbf{e}}_{\mathrm{x}} \\
& -Y_{m}^{\mathrm{e}, i}\left(\varrho, z_{n}^{\mathrm{e}, \mathrm{Ti}}\right) \sin [(m+1) \varphi] \widehat{\mathbf{e}}_{\mathrm{y}}
\end{aligned}
$$

$$
\begin{aligned}
\mathbf{A}_{m n}^{2 \mathrm{e}, i}(\mathbf{r}) & =Y_{m}^{\mathrm{e}, i}\left(\varrho, z_{n}^{\mathrm{e}, \mathrm{Ti}}\right) \sin [(m+1) \varphi] \widehat{\mathbf{e}}_{\mathrm{x}} \\
& +Y_{m}^{\mathrm{e}, i}\left(\varrho, z_{n}^{\mathrm{e}, \mathrm{Ti}}\right) \cos [(m+1) \varphi] \widehat{\mathbf{e}}_{\mathrm{y}}
\end{aligned}
$$

$\mathbf{A}_{m n}^{3 \mathrm{e}, i}(\mathbf{r})=Y_{0}^{\mathrm{e}, i}\left(\varrho, z_{n}^{\mathrm{e}, \mathrm{Ti}}\right) \widehat{\mathbf{e}}_{\mathrm{z}}$,

where

$Y_{m}^{\mathrm{e}}\left(\varrho, z_{n}^{\mathrm{e}}\right)=h_{m}^{(2)}\left(k_{\mathrm{e}} R_{z_{n}^{\mathrm{e}}}\right)\left(\frac{\rho}{R_{z_{n}^{\mathrm{e}}}}\right)^{m}$

$Y_{m}^{i}\left(\varrho, z_{n}^{\mathrm{Ti}}\right)=j_{m}\left(k_{\mathrm{T}} R_{z_{n}^{\mathrm{Ti}}}\right)\left(\frac{\rho}{R_{z_{n}^{\mathrm{Ti}}}}\right)^{m}$

$k_{\mathrm{T}}=k_{0} \sqrt{\varepsilon}, \varrho=(\rho, z), \rho^{2}=x^{2}+y^{2}$, and

$R_{z_{n}}^{2}=\rho^{2}+\left(z-z_{n}\right)^{2}$, while for longitudinal field approximation, the discrete sources are constructed from the scalar potentials $\Psi_{m n}$ and $\Psi_{n}$ as given below. For a P-polarized excitation, the approximate solution is given by

$$
\begin{aligned}
\mathbf{E}_{\mathrm{e}, \mathrm{Ti}}^{\mathcal{N}}(\mathbf{r}) & =\sum_{m=0}^{M} \sum_{n=1}^{N_{\mathrm{e}, \mathrm{Ti}}^{m}} p_{m n}^{\mathrm{e}, \mathrm{Ti}} \frac{\mathrm{j}}{k_{0} \varepsilon_{\mathrm{e}, i}} \nabla \times \nabla \times \mathbf{A}_{m n}^{1 \mathrm{e}, i}(\mathbf{r}) \\
& +q_{m n}^{\mathrm{e}, \mathrm{Ti}} \frac{\mathrm{j}}{\varepsilon_{\mathrm{e}, i}} \nabla \times \mathbf{A}_{m n}^{2 \mathrm{e}, i}(\mathbf{r}) \\
& +\sum_{n=1}^{N_{\mathrm{e}, T i}^{0}} r_{n}^{\mathrm{e}, \mathrm{Ti}} \frac{\mathrm{j}}{k_{0} \varepsilon_{\mathrm{e}, i}} \nabla \times \nabla \times \mathbf{A}_{n}^{3 \mathrm{e}, i}(\mathbf{r}), \\
\mathbf{E}_{\mathrm{L} i}^{\mathcal{N}}(\mathbf{r})= & \sum_{m=0}^{M} \sum_{n=1}^{N_{\mathrm{L} i}^{m}} p_{m n}^{\mathrm{L} i} \nabla \Psi_{m n}(\mathbf{r})+\sum_{n=1}^{N_{\mathrm{L} i}^{0}} r_{n}^{\mathrm{L} i} \nabla \Psi_{n}(\mathbf{r}), \\
\mathbf{H}_{\mathrm{e}, i}^{\mathcal{N}}(\mathbf{r}) & =\frac{\mathrm{j}}{k_{0}} \nabla \times \mathbf{E}_{\mathrm{e}, T i}^{\mathcal{N}}(\mathbf{r}),
\end{aligned}
$$

where the scalar potentials $\Psi_{m n}$ and $\Psi_{n}$ are defined respectively by

$\Psi_{m n}(\mathbf{r})=j_{m+1}\left(k_{\mathrm{L}} R_{z_{n}^{\mathrm{Li}}}\right) \cos [(m+1) \varphi]$,

$\Psi_{n}(\mathbf{r})=j_{0}\left(k_{\mathrm{L}} R_{z_{n}^{\mathrm{Li}}}\right)$,

$k_{\mathrm{L}}^{2}=\varepsilon / \xi^{2}$, and $M$ is the maximum number of azimuthal modes. For an S-polarized excitation, we have

$$
\begin{aligned}
\mathbf{E}_{\mathrm{e}, \mathrm{N} i}^{\mathcal{N}}(\mathbf{r}) & =\sum_{m=0}^{M} \sum_{n=1}^{N_{\mathrm{e}, T i}^{m}} p_{m n}^{\mathrm{e}, \mathrm{Ti}} \frac{\mathrm{j}}{k_{0} \varepsilon_{\mathrm{e}, i}} \nabla \times \nabla \times \mathbf{A}_{m n}^{2 \mathrm{e}, i}(\mathbf{r}) \\
& +q_{m n}^{\mathrm{e}, \mathrm{Ti}} \frac{\mathrm{j}}{\varepsilon_{\mathrm{e}, i}} \nabla \times \mathbf{A}_{m n}^{1 \mathrm{e}, i}(\mathbf{r}) \\
& +\sum_{n=1}^{N_{\mathrm{e}, T i}^{0}} r_{n}^{\mathrm{e}, \mathrm{Ti}} \frac{\mathrm{j}}{\varepsilon_{\mathrm{e}, i}} \nabla \times \mathbf{A}_{n}^{3 \mathrm{e}, i}(\mathbf{r}), \\
\mathbf{E}_{\mathrm{L} i}^{\mathcal{N}}(\mathbf{r})= & \sum_{m=0}^{M} \sum_{n=1}^{N_{\mathrm{Li}}^{m}} p_{m n}^{\mathrm{L} i} \nabla \Psi_{m n}(\mathbf{r}), \\
\mathbf{H}_{\mathrm{e}, i}^{\mathcal{N}}(\mathbf{r}) & =\frac{\mathrm{j}}{k_{0}} \nabla \times \mathbf{E}_{\mathrm{e}, T i}^{\mathcal{N}}(\mathbf{r}),
\end{aligned}
$$

where the scalar potential $\Psi_{m n}$ is now defined by

$\Psi_{m n}(\mathbf{r})=j_{m+1}\left(k_{\mathrm{L}} R_{z_{n}^{\mathrm{Li}}}\right) \sin [(m+1) \varphi]$.

Some remarks are in order:

1. $\mathcal{N}$ is a multi-index incorporating both the maximum number of azimuthal modes $M$, and the number of discrete sources $N_{\mathrm{e}}^{m}$, $N_{\mathrm{T} i}^{m}$ and $N_{\mathrm{L} i}^{m}$.

2. From (28) and (33) it is apparent that only the transverse field $\mathbf{E}_{\mathrm{T} i}$ contributes to the magnetic field $\mathbf{H}_{i}$ inside particle $i$.

3. In the case of an S-polarized excitation, the azimuthal independent harmonic does not contain the nonlocal term $\nabla \Psi_{n}(\mathbf{r})$ appearing in (27). The reason is that in this case, there is no normal component of the electric field, and so, the additional boundary condition is not required.

As the electromagnetic fields given by (26)-(28) and (31)-(33) solve the Maxwell equations (6) and (7), we have to determine the amplitudes of discrete sources

$\left\{p_{m n}^{\mathrm{e}, \mathrm{Ti}}, p_{m n}^{\mathrm{L} i}, q_{m n}^{\mathrm{e}, \mathrm{Ti}}, r_{n}^{\mathrm{e}, \mathrm{Ti}}, r_{n}^{\mathrm{L} i}\right\}$ 
such that the boundary conditions (8)-(10) are fulfilled. By means of the generalized point matching technique, we obtain the following relations for amplitudes computation:

$$
\begin{aligned}
& \widehat{\mathbf{n}}_{i} \times \int_{0}^{2 \pi}\left[\mathbf{E}_{\mathrm{T} i}\left(\varrho_{l}, \varphi\right)+\mathbf{E}_{\mathrm{L} i}\left(\varrho_{l}, \varphi\right)-\mathbf{E}_{\mathrm{e}}\left(\varrho_{l}, \varphi\right)\right] \mathrm{e}^{-\mathrm{j} m \varphi} \mathrm{d} \varphi \\
& =\widehat{\mathbf{n}}_{i} \times \int_{0}^{2 \pi} \mathbf{E}_{0}\left(\varrho_{l}, \varphi\right) \mathrm{e}^{-\mathrm{j} m \varphi} \mathrm{d} \varphi,
\end{aligned}
$$

$\widehat{\mathbf{n}}_{i} \times \int_{0}^{2 \pi}\left[\mathbf{H}_{i}\left(\varrho_{l}, \varphi\right)-\mathbf{H}_{\mathrm{e}}\left(\varrho_{l}, \varphi\right)\right] \mathrm{e}^{-\mathrm{j} m \varphi} \mathrm{d} \varphi$

$=\widehat{\mathbf{n}}_{i} \times \int_{0}^{2 \pi} \mathbf{H}_{0}\left(\varrho_{l}, \varphi\right) \mathrm{e}^{-\mathrm{j} m \varphi} \mathrm{d} \varphi$,

$\widehat{\mathbf{n}}_{i} \cdot \int_{0}^{2 \pi}\left[\varepsilon_{\mathrm{b}} \mathbf{E}_{\mathrm{Ti}}\left(\varrho_{l}, \varphi\right)+\varepsilon_{\mathrm{b}} \mathbf{E}_{\mathrm{Li}}\left(\varrho_{l}, \varphi\right)-\varepsilon_{\mathrm{e}} \mathbf{E}_{\mathrm{e}}\left(\varrho_{l}, \varphi\right)\right] \mathrm{e}^{-\mathrm{j} m \varphi} \mathrm{d} \varphi$

$=\widehat{\mathbf{n}}_{i} \cdot \int_{0}^{2 \pi} \varepsilon_{\mathrm{e}} \mathbf{E}_{0}\left(\varrho_{l}, \varphi\right) \mathrm{e}^{-\mathrm{j} m \varphi} \mathrm{d} \varphi$,

where $\left\{\varrho_{l}\right\}_{l=1}^{L}$ is the set of matching points distributed in the azimuthal plane $\varphi=$ const. For each $m$, the numbers of discrete sources and matching points are chosen as

$2 N_{\mathrm{e}}^{m}+2 N_{\mathrm{T} i}^{m}+N_{\mathrm{L} i}^{m}<5 L$,

in which case, we are led to an overdetermined system of equations for amplitudes determination. The system of equations is solved by QR matrix factorization for a given set of incident angles $\theta_{0}$, and simultaneously, for P- and S-polarized excitations.

Once the amplitudes of discrete sources are known, the components of the far-field pattern

$\mathbf{F}(\theta, \varphi)=F_{\theta}(\theta, \varphi) \widehat{\mathbf{e}}_{\vartheta}+F_{\varphi}(\theta, \varphi) \widehat{\mathbf{e}}_{\varphi}$,

defined through the relation

$\mathbf{E}_{\mathrm{e}}(\mathbf{r})=\frac{\mathrm{e}^{-\mathrm{j} k_{\mathrm{e}} r}}{r} \mathbf{F}(\theta, \varphi)+o\left(\frac{1}{r}\right), \quad r \rightarrow \infty$,

are computed for a P-polarized excitation as

$$
\begin{aligned}
F_{\theta}(\theta, \varphi)= & \mathrm{j} k_{\mathrm{e}} \sum_{m=0}^{M}(\mathrm{j} \sin \theta)^{m} \cos [(m+1) \varphi] \\
& \times \sum_{n=1}^{N_{\mathrm{e}}^{m}}\left(p_{m n}^{\mathrm{e}} \cos \theta+q_{m n}^{\mathrm{e}}\right) \mathrm{e}^{-\mathrm{j} k_{\mathrm{e}} z_{n}^{\mathrm{e}} \cos \theta} \\
& -\mathrm{j} k_{\mathrm{e}} \sin \theta \sum_{n=1}^{N_{\mathrm{e}}^{0}} r_{n}^{\mathrm{e}} \mathrm{e}^{-\mathrm{j} k_{\mathrm{e}} z_{n}^{\mathrm{e}} \cos \theta}, \\
F_{\varphi}(\theta, \varphi)= & -\mathrm{j} k_{\mathrm{e}} \sum_{m=0}^{M}(\mathrm{j} \sin \theta)^{m} \sin [(m+1) \varphi] \\
& \times \sum_{n=1}^{N_{\mathrm{e}}^{m}}\left(p_{m n}^{\mathrm{e}}+q_{m n}^{\mathrm{e}} \cos \theta\right) \mathrm{e}^{-\mathrm{j} k_{\mathrm{e}} z_{n}^{\mathrm{e}} \cos \theta}
\end{aligned}
$$

and for an S-polarized excitation as

$$
\begin{aligned}
& F_{\theta}(\theta, \varphi)= \mathrm{j} k_{\mathrm{e}} \sum_{m=0}^{M}(\mathrm{j} \sin \theta)^{m} \sin [(m+1) \varphi] \\
& \times \sum_{n=1}^{N_{\mathrm{e}}^{m}}\left(p_{m n}^{\mathrm{e}} \cos \theta-q_{m n}^{\mathrm{e}}\right) \mathrm{e}^{-\mathrm{j} k_{\mathrm{e}} z_{n}^{\mathrm{e}} \cos \theta} \\
& F_{\varphi}(\theta, \varphi)=\mathrm{j} k_{\mathrm{e}} \sum_{m=0}^{M}(\mathrm{j} \sin \theta)^{m} \cos [(m+1) \varphi]
\end{aligned}
$$

$$
\begin{aligned}
& \times \sum_{n=1}^{N_{\mathrm{e}}^{m}}\left(p_{m n}^{\mathrm{e}} \cos \theta-q_{m n}^{\mathrm{e}}\right) \mathrm{e}^{-\mathrm{j} k_{\mathrm{e}} z_{n}^{\mathrm{e}} \cos \theta} \\
& +\mathrm{j} k_{\mathrm{e}} \sin \theta \sum_{n=1}^{N_{\mathrm{e}}^{0}} r_{n}^{\mathrm{e}} \mathrm{e}^{-\mathrm{j} k_{\mathrm{e}} z_{n}^{\mathrm{e}} \cos \theta} .
\end{aligned}
$$

The differential scattering cross section is then calculated as

$\sigma(\theta, \varphi)=\left|F_{\theta}(\theta, \varphi)\right|^{2}+\left|F_{\varphi}(\theta, \varphi)\right|^{2}$,

the scattering cross section as

$\sigma_{\mathrm{sct}}=\int_{0}^{2 \pi} \int_{0}^{\pi} \sigma(\theta, \varphi) \sin \theta \mathrm{d} \theta \mathrm{d} \varphi$,

and the extinction cross section for a P- and an S-polarized excitation as

$\sigma_{\text {ext }}=-\frac{4 \pi}{k_{\mathrm{e}}} \operatorname{Im}\left[F_{\theta}\left(\pi-\theta_{0}, \pi\right)\right]$

and

$\sigma_{\text {ext }}=\frac{4 \pi}{k_{\mathrm{e}}} \operatorname{Im}\left[F_{\varphi}\left(\pi-\theta_{0}, \pi\right)\right]$,

respectively. From (41)-(44) it is readily seen that the components of the far-field pattern are expressed through finite linear combinations of elementary functions, and so, that no integration procedure is required for their computation. Besides, the errors in the solution can be estimated by computing the residual norm of the surface fields at the particle surfaces $\partial D_{i}, i=1,2$.

\section{Numerical analysis}

In this section we analyze the scattering of a plasmonic dimer consisting of two identical metallic prolate spheroids. Each prolate spheroid has an equivolume diameter of $D_{\mathrm{p}}=16 \mathrm{~nm}$. The material of the spheroids is gold $(\mathrm{Au})$ or silver $(\mathrm{Ag})$. For these materials, the frequency dependent refractive indices $n_{i}=\sqrt{\varepsilon_{i}}=\sqrt{\varepsilon}$ are taken from [32]. The corresponding GNOR parameters are [12]:

$\hbar \omega_{\mathrm{p}}=9.02 \mathrm{eV}, \quad \hbar \gamma=0.071 \mathrm{eV}, \quad v_{\mathrm{F}}=1.39 \mu \mathrm{m} / \mathrm{s}$,

$D=1.90 \times 10^{8} \mu \mathrm{m}^{2} / \mathrm{s}$

for $\mathrm{Au}$, and

$\hbar \omega_{\mathrm{p}}=8.99 \mathrm{eV}, \quad \hbar \gamma=0.025 \mathrm{eV}, \quad v_{\mathrm{F}}=1.39 \mu \mathrm{m} / \mathrm{s}$,

$D=3.61 \times 10^{8} \mu \mathrm{m}^{2} / \mathrm{s}$

for Ag. In [33], it has been found that for this scattering problem, the plasmonic resonance seems to be more pronounced for a $\mathrm{P}$ polarized excitationand the incident angle $\theta_{0}=90^{\circ}$, when the incident electric field $\mathbf{E}_{0}$ is parallel to the $O z$ axis (the axis of symmetry of the prolate spheroids). For this reason, the incident angle is chosen as $\theta_{0}=90^{\circ}$. For the physical reason of this choice the interested reader is referredto Nordlander et al. [34].

The main numerical problem of simulating the nonlocal response is the large difference between the longitudinal and transverse wavenumbers $k_{\mathrm{L}}$ and $k_{\mathrm{T}}$, respectively. The results illustrated in Fig. 2 show that $k_{\mathrm{L}}$ exceeds $k_{\mathrm{T}}$ by about two order of magnitudes. Therefore,

1. the number of discrete sources for the longitudinal field $N_{\mathrm{L} i}^{m}$ is approximately three to four times larger than that for the transverse field $N_{\mathrm{T} i}^{m}$,

2. the number of matching points $L$ is ten times larger than that used in the local-response approximation (leading to a discretization step of about $0.1 \mathrm{~nm}$ or less).

In Fig. 3, we plot the extinction and scattering cross-sections computed by means of LRA and GNOR models for a gold dimer. The half-axes of the spheroid are $a=12.7 \mu \mathrm{m}$ and $b=25.4 \mu \mathrm{m}$, 


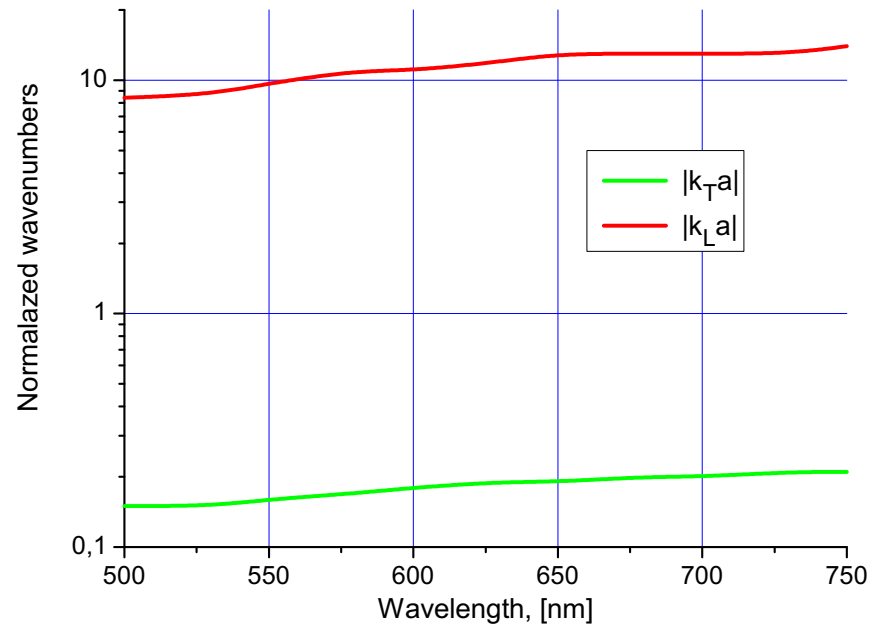

Fig. 2. Absolute values of the normalized wavenumbers $k_{\mathrm{L}} a$ and $k_{\mathrm{T}} a$, where $a$ is the minor axis of the spheroid.

and the gap size $d=1 \mathrm{~nm}$. The background medium is water $\left(n_{\mathrm{e}}=\right.$ $\left.\sqrt{\varepsilon_{\mathrm{e}}}=1.33\right)$ and glass $\left(n_{\mathrm{e}}=\sqrt{\varepsilon_{\mathrm{e}}}=1.52\right)$. The results show a blue shift and a plasmon mode damping for both background media, and a larger difference between the extinction and scattering cross sections, and so, a larger absorption cross section, in the case of GNOR than in the case of LRA.

The variations of the extinction and scattering cross-sections with respect to the aspect ratio $r=b / a$ and the gap size $d$ are illustrated in Figs. 4 and 5. The material of the particles is gold and the background medium is water. As before, a blue shift, a plasmon mode damping, and a plasmon mode broadening are visible for both cross sections. Besides, the blue shift, the plasmon mode damping, and the plasmon mode broadening increase when the aspect ratio $r$ increases, and the gap size $d$ decreases. The plots in Fig. 6 demonstrate that the electric field intensity in the center point in between of the spheroids increases when the spheroids approach each other. Besides based on further simulations we did not find any shift between the far and near field intensity which is in agreement with paper [38].

The physical predictions of a nonlocal response model are sensitive to the correct implementation of the additional boundary condition [35]. In Fig. 7 we illustrate a comparison of the results obtained by using the additional boundary condition (cf. (10))

$$
\varepsilon_{\mathrm{b}} \widehat{\mathbf{n}}_{i} \cdot \mathbf{E}_{i}\left(\boldsymbol{r}_{l}\right)=\varepsilon_{\mathrm{e}} \widehat{\mathbf{n}}_{i} \cdot\left[\mathbf{E}_{\mathrm{e}}\left(\boldsymbol{r}_{l}\right)+\mathbf{E}_{0}\left(\boldsymbol{r}_{l}\right)\right],
$$

based on the continuity of the normal component of the free current density, and an additional boundary condition based on the continuity of the normal component of the electric field [36,37], that is,

$\widehat{\mathbf{n}}_{i}\left(\mathbf{r}_{l}\right) \cdot \mathbf{E}_{i}\left(\mathbf{r}_{l}\right)=\widehat{\mathbf{n}}_{i}\left(\mathbf{r}_{l}\right) \cdot\left[\mathbf{E}_{\mathrm{e}}\left(\mathbf{r}_{l}\right)+\mathbf{E}_{0}\left(\mathbf{r}_{l}\right)\right]$.

The plots show that the blue shift and the plasmon mode damping are considerably different; they are more pronounced for (50) than for (49). For a detailed discussion of the physical meaning of boundary conditions (49) and (50) we refer to [35]. On the other hand, in the framework of LRA, the continuity of the tangential components of the electric and magnetic fields yields the continuity of the normal component of the displacement field

$\varepsilon_{i} \widehat{\mathbf{n}}_{i}\left(\mathbf{r}_{l}\right) \cdot \mathbf{E}_{i}\left(\mathbf{r}_{l}\right)=\varepsilon_{\mathrm{e}} \widehat{\mathbf{n}}_{i}\left(\mathbf{r}_{l}\right) \cdot\left[\mathbf{E}_{\mathrm{e}}\left(\mathbf{r}_{l}\right)+\mathbf{E}_{0}\left(\mathbf{r}_{l}\right)\right]$.

An interesting exercise is to use a nonlocal response model in which the LRA boundary condition (51) is used as additional boundary condition. The results illustrated in Fig. 8 show that in the case of boundary condition (51), a smaller plasmon mode damping is present and that no blue shift is visible. This is a consequence of the fact that the use of the boundary condition (51) reduces the nonlocal screening which appears to be the main origin for the blue shift [7].

\section{Conclusions}

A modified scheme of the discrete sources method based on the generalized nonlocal optical response model has been applied to analyze the scattering by a plasmonic dimer consisting of two identical axisymmetric metallic particles.In the new scheme, the discrete sources for the transverse field (curl and double curl of vector potentials) and for the longitudinal field (gradient of scalar potentials) approximation are constructed based on the system of lowest order distributed multipoles [39]. Because the longitudinal wavenumber exceeds the transverse wavenumber by about two order of magnitude, special attention has been payed to the numerical implementation of the discrete sources model. In this context, it has been found that the number of matching points should be at least ten times larger than that used in the local-response approximation.

The extinction and scattering cross sections of a plasmonic dimer consisting of two identical metallic prolate spheroids have
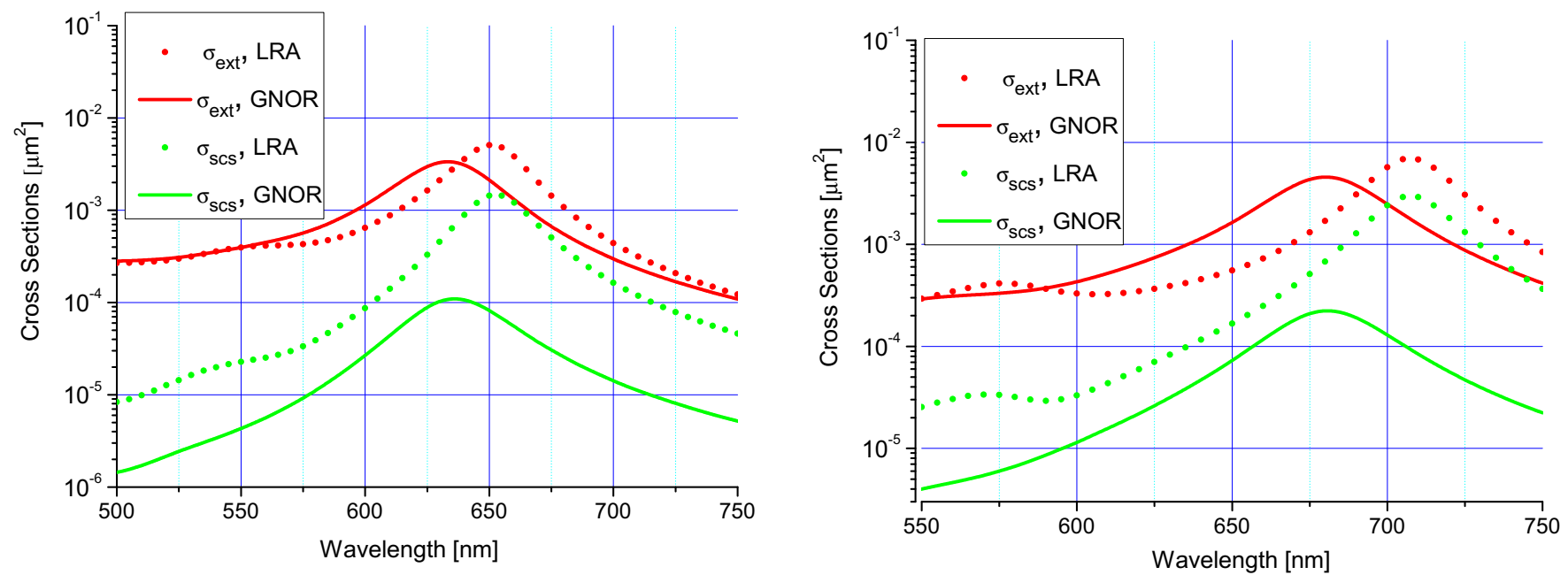

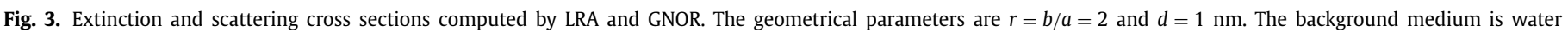
(left) and glass (right). 

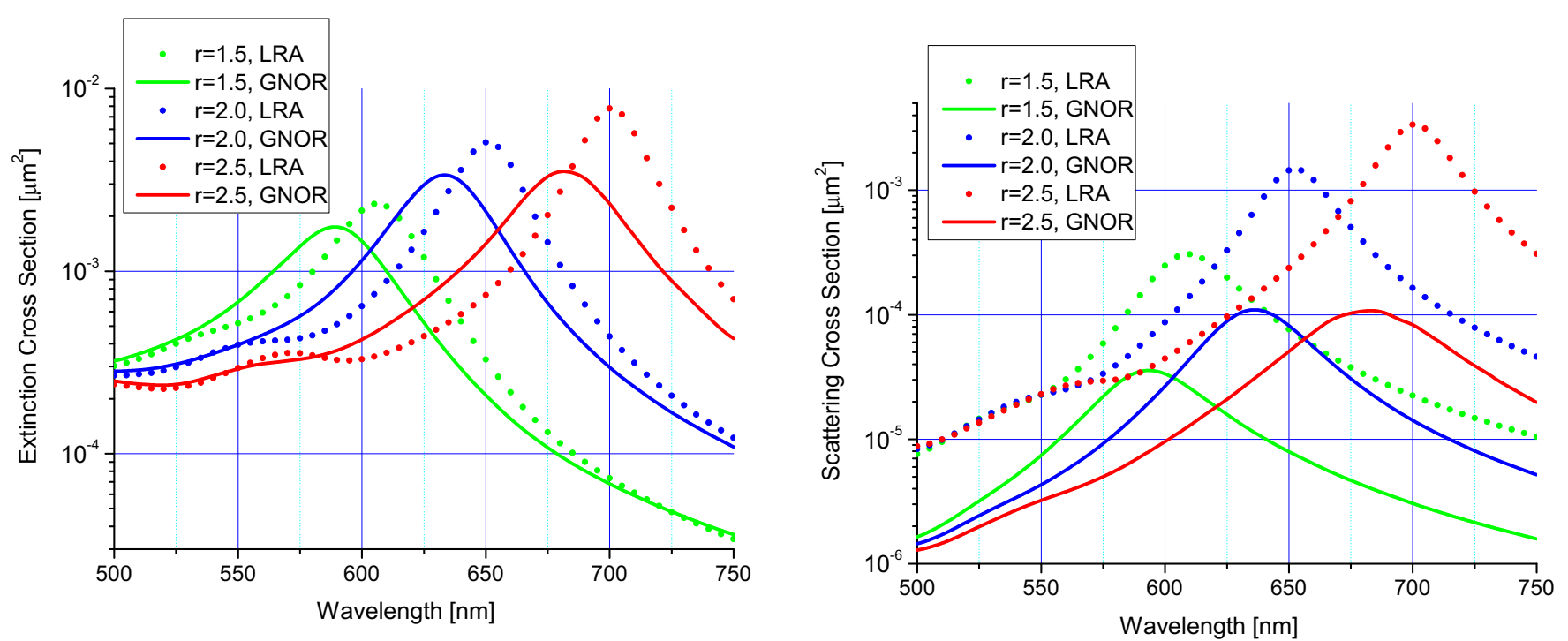

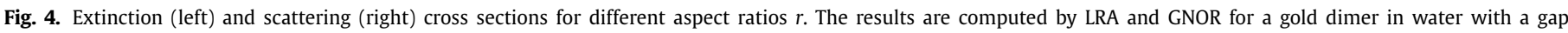
size $d=1 \mathrm{~nm}$.
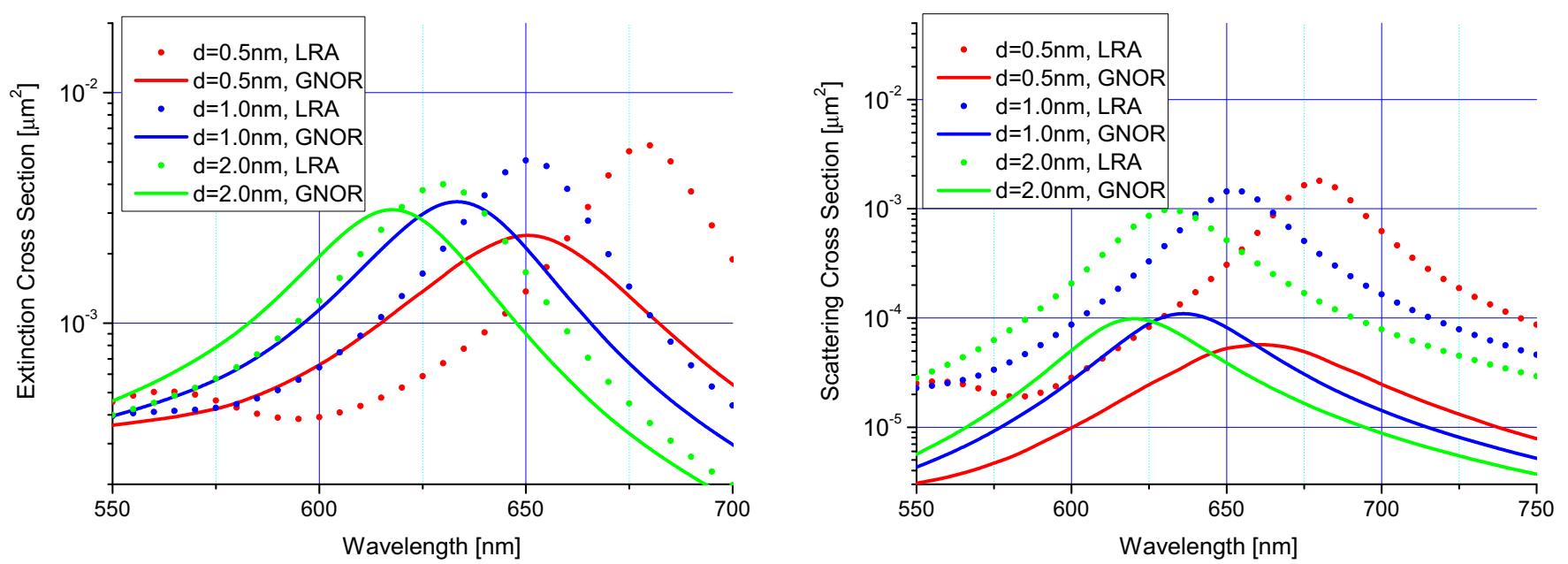

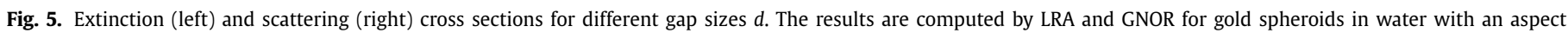
ratio $r=2$.

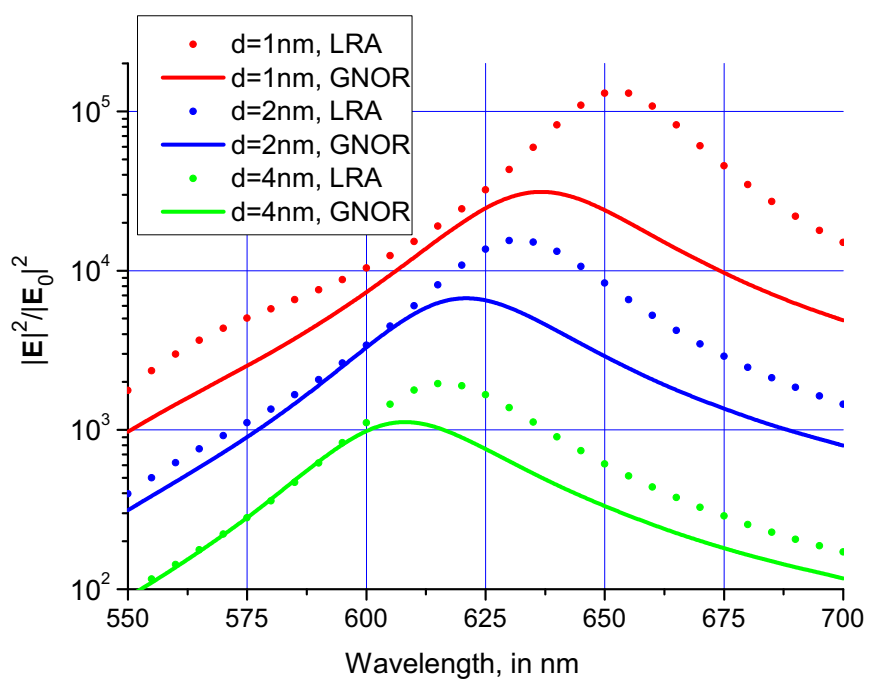

Fig. 6. Total field intensity $|E|^{2} /\left|E_{0}\right|^{2}$ in the center of the gap between spheroids for different gap sizes. The results are computed by LRA and GNOR for Au spheroids in water with an aspect ratio $r=2$. been computed in the frequency domain for different spheroid aspect ratios and gap sizes. Comparing the results obtained by the discrete sources method with the generalized nonlocal optical response model and those with the local-response approximation, a blue shift, a plasmon mode damping, and a plasmon mode broadening of the optical cross sections has been evidenced. These effects are more pronounced under the deformation of the constituent spheroids when the aspect ratio of the spheroid increases and the gap size decreases. This is in agreement with the results of Filter et al. [40] where dimer consisted of small plasmonic particles (size $6.7 \mathrm{~nm}$ ) of different shapes excited by a vertical electric dipole located inside the gap was investigated based on COMSOL application.

A significant difference in the optical cross sections has been found when using as additional boundary condition the continuity of the normal component of the free current density and the continuity of the normal component of the electric field. These results emphasizes that a correct implementation of the additional boundary condition is absolutely demanding. 

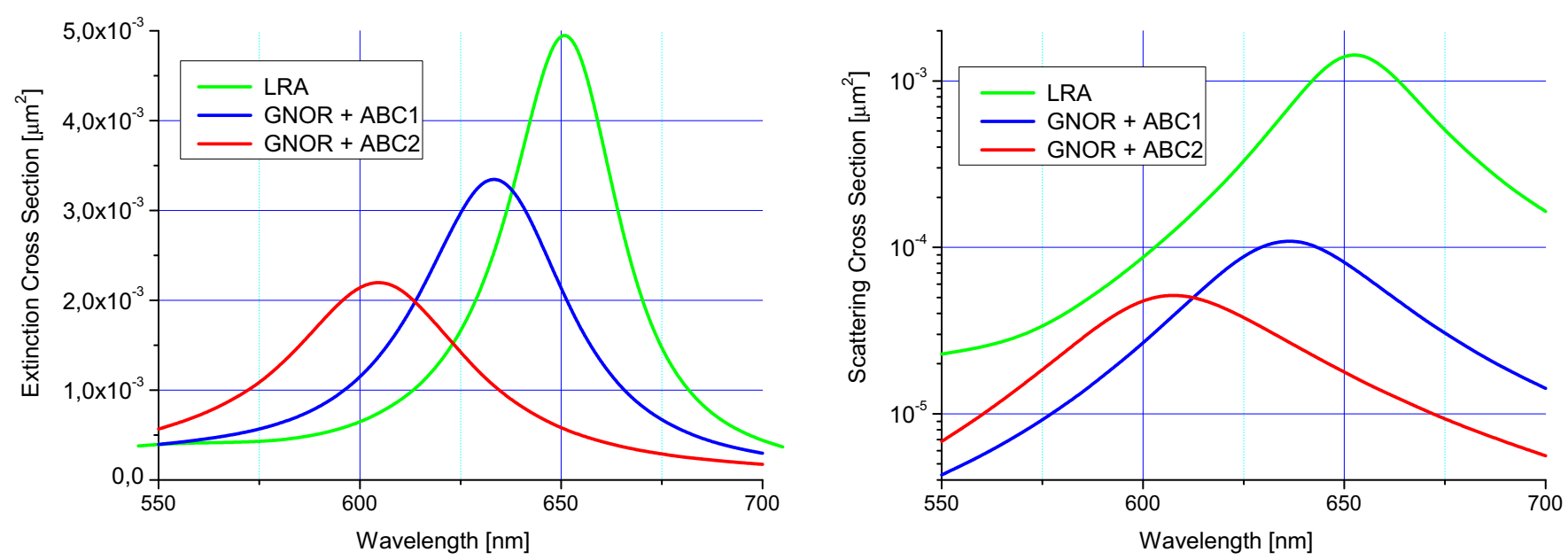

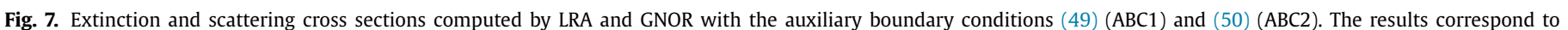
a gold dimer in water with an aspect ratio $r=2$ and a gap size $d=1 \mathrm{~nm}$.
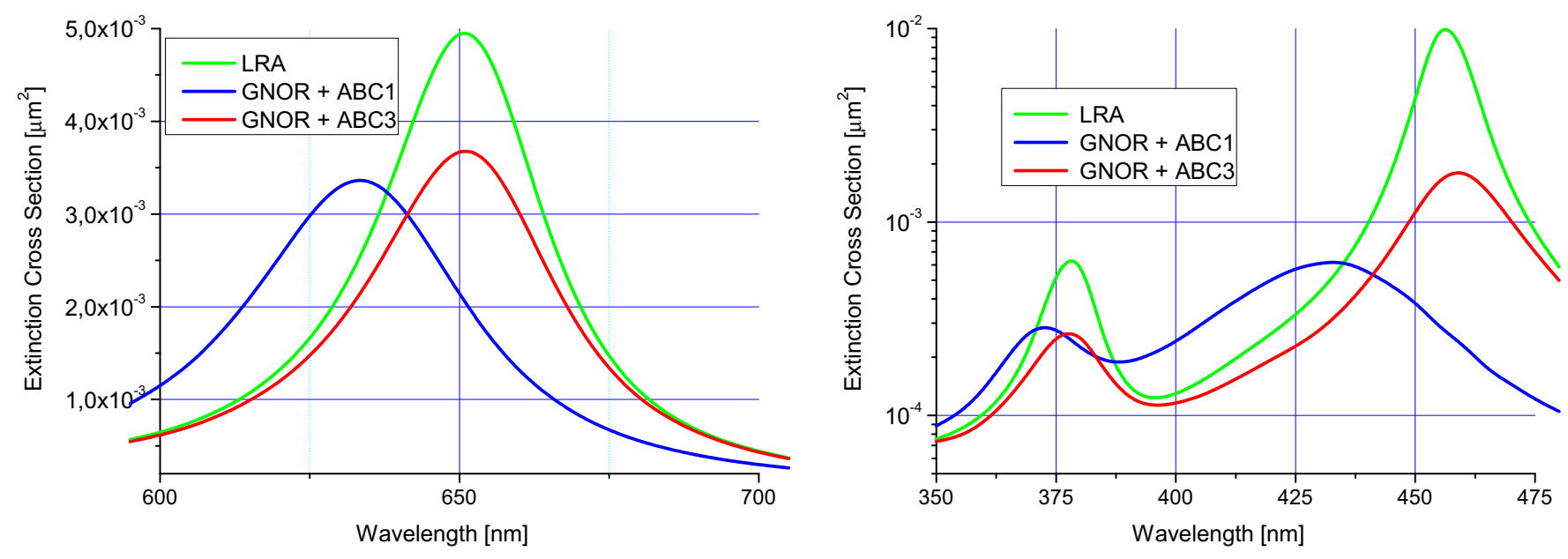

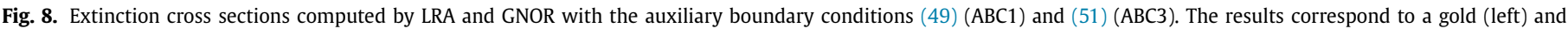
a silver (right) dimer in water with an aspect ratio $r=2$ and a gap size $d=1 \mathrm{~nm}$.

\section{Acknowledgment}

This work was supported by the Deutsche Forschungsgemeinschaft (DFG) under grant WR 22/59-1.

\section{Appendix. Nonlocal response theories}

In this appendix we review the nonlocal response theories by closely following the analysis given in [12,35]. The constitutive relation relating the displacement field $\mathbf{D}$ and the electric field $\mathbf{E}$ is

$\mathbf{D}(\mathbf{r}, \omega)=\varepsilon_{0} \int \varepsilon\left(\mathbf{r}, \mathbf{r}^{\prime}, \omega\right) \mathbf{E}\left(\mathbf{r}^{\prime}, \omega\right) \mathrm{d}^{3} \mathbf{r}$,

where $\omega$ is the frequency, $\varepsilon_{0}$ the vacuum permittivity, and $\varepsilon(\mathbf{r}$, $\left.\mathbf{r}^{\prime}, \omega\right)$ the (scalar) nonlocal permittivity of the metal. In the localresponse approximation, nonlocal effects are neglected; we have $\varepsilon\left(\mathbf{r}, \mathbf{r}^{\prime}, \omega\right)=\delta\left(\mathbf{r}-\mathbf{r}^{\prime}\right) \varepsilon(\omega)$, giving

$\mathbf{D}(\mathbf{r}, \omega)=\varepsilon_{0} \varepsilon(\omega) \mathbf{E}(\mathbf{r}, \omega)$,

where $\varepsilon(\omega)$ is the spatially independent permittivity. In a nonlocal response model, the vector wave equation for the electric field is given by

$\nabla \times \nabla \times \mathbf{E}(\mathbf{r}, \omega)=\left(\frac{\omega}{c}\right)^{2} \frac{1}{\varepsilon_{0}} \mathbf{D}(\mathbf{r}, \omega)$

$$
=\left(\frac{\omega}{c}\right)^{2} \int \varepsilon\left(\mathbf{r}, \mathbf{r}^{\prime}, \omega\right) \mathbf{E}\left(\mathbf{r}^{\prime}, \omega\right) \mathrm{d}^{3} \mathbf{r},
$$

where $c=1 / \sqrt{\varepsilon_{0} \mu_{0}}$ is the speed of light in vacuum. The nonlocal permittivity is written as

$\varepsilon\left(\mathbf{r}, \mathbf{r}^{\prime}, \omega\right)=\delta\left(\mathbf{r}-\mathbf{r}^{\prime}\right) \varepsilon(\omega)+f\left(\mathbf{r}-\mathbf{r}^{\prime}, \omega\right)$,

where $f\left(\mathbf{r}-\mathbf{r}^{\prime}, \omega\right)$ is a scalar nonlocal response function. The nonlocal response function is assumed to be symmetric and shortranged, which means that

$\int f(\mathbf{r}, \omega) \mathbf{r} \mathrm{d}^{3} \mathbf{r}=0$ and $\int f(\mathbf{r}, \omega) \mathbf{r}^{2} \mathrm{~d}^{3} \mathbf{r}=2 \xi^{2}$,

where $\mathbf{r}^{2}=x^{2} \widehat{\mathbf{e}}_{\mathrm{x}}+y^{2} \widehat{\mathbf{e}}_{\mathrm{y}}+z^{2} \widehat{\mathbf{e}}_{\mathrm{z}}, \quad\left(\widehat{\mathbf{e}}_{\mathrm{x}}, \widehat{\mathbf{e}}_{\mathrm{y}}, \widehat{\mathbf{e}}_{\mathrm{z}}\right)$ are the Cartesian unit vectors, and $\xi$ is the length scale of the nonlocal response function. Under this assumption and by considering a second-order Taylor expansion of $\mathbf{E}\left(\mathbf{r}^{\prime}, \omega\right)$ around $\mathbf{r}$, the integral in (54) can be computed analytically; the vector wave equation (54) then takes the form

$\nabla \times \nabla \times \mathbf{E}(\mathbf{r}, \omega)=\left(\frac{\omega}{c}\right)^{2}\left[\varepsilon(\omega)+\xi^{2} \triangle\right] \mathbf{E}(\mathbf{r}, \omega)$.

From (56) it is apparent that the scalar nonlocal response is reproduced by the Laplacian term in the vector wave equation. The hydrodynamical model and the generalized nonlocal optical response model are nonlocal response theories leading to a vector wave 
equation as in (56), that give simple computational formulas for the length scale of the nonlocal response function $\xi$.

In the hydrodynamical model for the free-electron gas, the energy of the electron plasma is expressed in terms of the electron density $n(\mathbf{r}, t)$ and the velocity field $\mathbf{v}(\mathbf{r}, t)$. The dynamics of $n(\mathbf{r}$, $t)$ and $\mathbf{v}(\mathbf{r}, t)$ due to the electric field $\mathbf{E}(\mathbf{r}, t)$ are obtained by differentiating the energy with respect to these variables. Differentiation of the energy with respect to the velocity field and electron density gives the hydrodynamic equation of motion and the continuity equation, respectively. These equations are solved by using a linearization approach; the physical fields are expanded in a nonoscillating term and a (small) first-order dynamic term. In the frequency domain, we are led to the vector wave equation

$\nabla \times \nabla \times \mathbf{E}(\mathbf{r}, \omega)=\left(\frac{\omega}{c}\right)^{2} \varepsilon_{\mathrm{b}} \mathbf{E}(\mathbf{r}, \omega)-\mathbf{j} \omega \mu_{0} \mathbf{J}(\mathbf{r}, \omega)$

and the constitutive relation for the current density

$\left(\frac{\beta^{2}}{\omega^{2}-j \gamma \omega}\right) \nabla[\nabla \cdot \mathbf{J}(\mathbf{r}, \omega)]+\mathbf{J}(\mathbf{r}, \omega)=\sigma(\omega) \mathbf{E}(\mathbf{r}, \omega)$.

Here, $\mathbf{J}(\mathbf{r}, \omega)=-e n_{0} \mathbf{v}(\mathbf{r}, \omega)$ is the current density due to free charges, $n_{0}$ the equilibrium electron density of the free electrons, $\beta^{2}=(3 / 5) v_{\mathrm{F}}^{2}, v_{\mathrm{F}}$ the Fermi velocity, $\gamma$ the Drude damping rate,

$\omega_{\mathrm{p}}^{2}=\frac{n_{0} e^{2}}{\varepsilon_{0} m}$

the plasma frequency of the metal,

$\sigma(\omega)=-\mathrm{j} \varepsilon_{0} \frac{\omega_{\mathrm{p}}^{2}}{\omega-\mathrm{j} \gamma}$

the Drude conductivity, related to the Drude permittivity $\varepsilon(\omega)$ by

$\varepsilon(\omega)=\varepsilon_{\mathrm{b}}-\mathrm{j} \frac{\sigma(\omega)}{\varepsilon_{0} \omega}=\varepsilon_{\mathrm{b}}-\frac{\omega_{\mathrm{p}}^{2}}{\omega^{2}-\mathrm{j} \gamma \omega}$,

and $\varepsilon_{\mathrm{b}}$ the permittivity associated to bound charges (ions and electrons). Obviously, in the limit $\beta \rightarrow 0$, i.e., $v_{\mathrm{F}} \rightarrow 0$, the constitutive relation (58) simplifies to Ohm's law $\mathbf{J}=\sigma \mathbf{E}$.

The hydrodynamic model includes the convective current but neglects the currents due to diffusion. In the generalized nonlocal optical response model the hydrodynamic theory is extended to account for electron diffusion. The inclusion of electron diffusion changes the continuity equation, which in its linearized form is the convection-diffusion equation. In this model, the free current density includes a diffusion contribution

$\mathbf{J}(\mathbf{r}, \omega)=-e n_{0} \mathbf{v}(\mathbf{r}, \omega)+e D \nabla n(\mathbf{r}, \omega)$,

where $D$ is the diffusion constant, while the constitutive relation for the free current density is (compare with (58))

$\left(\frac{\beta^{2}}{\omega^{2}-\mathrm{j} \gamma \omega}+\mathrm{j} \frac{D}{\omega}\right) \nabla[\nabla \cdot \mathbf{J}(\mathbf{r}, \omega)]+\mathbf{J}(\mathbf{r}, \omega)=\sigma(\omega) \mathbf{E}(\mathbf{r}, \omega)$.

To derive a vector wave equation as in (56), the current density is eliminated from Eqs. (57) and (59). Applying the operator $\nabla(\nabla \cdot)$ to Eq. (57), accounting of the identity $\nabla \cdot(\nabla \times \mathbf{F})=0$, and inserting the result in (59) gives

$\mathbf{J}(\mathbf{r})=\sigma \mathbf{E}(\mathbf{r})+\mathrm{j} \omega \varepsilon_{0} \xi^{2} \nabla[\nabla \cdot \mathbf{E}(\mathbf{r})]$,

so that the vector wave equations (57) becomes

$\nabla \times \nabla \times \mathbf{E}(\mathbf{r}, \omega)=\left(\frac{\omega}{c}\right)^{2}\left[\varepsilon(\omega)+\xi^{2}(\omega) \nabla(\nabla \cdot)\right] \mathbf{E}(\mathbf{r}, \omega)$,

where $\xi$ is the length scale of the generalized nonlocal optical response model,

$\xi^{2}(\omega)=\varepsilon_{\mathrm{b}} \frac{\beta^{2}+D(\gamma+\mathrm{j} \omega)}{\omega^{2}-\mathrm{j} \gamma \omega}$
Obviously, the vector wave equation (61) is as in (56) but in which the Laplace operator is replaced by the gradient-of-divergence operator $\nabla(\nabla \cdot)$. In fact, the presence of the Laplace operator in (56) is a consequence of using the scalar nonlocal response function $f\left(\mathbf{r}-\mathbf{r}^{\prime}, \omega\right)$. From (54) and (61), it follows that in the nonlocal response theory, the displacement field incorporating the contributions of bound and free charges is given by

$\mathbf{D}(\mathbf{r}, \omega)=\varepsilon_{0}\left[\varepsilon(\omega)+\xi^{2}(\omega) \nabla(\nabla \cdot)\right] \mathbf{E}(\mathbf{r}, \omega)$.

Actually, the vector wave equation (61) is equivalent to the Maxwell equations for nonmagnetic media,

$\nabla \times \mathbf{E}(\mathbf{r}, \omega)=-\mathrm{j} \omega \mu_{0} \mathbf{H}(\mathbf{r}, \omega)$,

$\nabla \times \mathbf{H}(\mathbf{r}, \omega)=\mathrm{j} \omega \varepsilon_{0}\left[\varepsilon(\omega)+\xi^{2}(\omega) \nabla(\nabla \cdot)\right] \mathbf{E}(\mathbf{r}, \omega)$.

Usage of the transformations $\sqrt{\varepsilon_{0}} \mathbf{E}(\mathbf{r}, \omega) \rightarrow \mathbf{E}(\mathbf{r}, \omega)$ and $\sqrt{\mu_{0}} \mathbf{H}(\mathbf{r}, \omega) \rightarrow \mathbf{H}(\mathbf{r}, \omega)$, then yields the dimensionless form of the Maxwell equations

$\nabla \times \mathbf{E}(\mathbf{r}, \omega)=-\mathbf{j} k_{0} \mathbf{H}(\mathbf{r}, \omega)$,

$\nabla \times \mathbf{H}(\mathbf{r}, \omega)=\mathrm{j} k_{0}\left[\varepsilon(\omega)+\xi^{2}(\omega) \nabla(\nabla \cdot)\right] \mathbf{E}(\mathbf{r}, \omega)$,

where $k_{0}=\omega \sqrt{\varepsilon_{0} \mu_{0}}$ is the wavenumber in vacuum.

The electric field sums the contributions of a divergence-free transverse field and a curl-free longitudinal field, i.e.,

$\mathbf{E}(\mathbf{r}, \omega)=\mathbf{E}_{\mathrm{T}}(\mathbf{r}, \omega)+\mathbf{E}_{\mathrm{L}}(\mathbf{r}, \omega)$,

with

$\nabla \cdot \mathbf{E}_{\mathrm{T}}(\mathbf{r}, \omega)=0, \quad \nabla \times \mathbf{E}_{\mathrm{L}}(\mathbf{r}, \omega)=0$.

Inserting (68) in (61) and using the identity $\nabla \times \nabla \times \mathbf{F}=\nabla(\nabla$. F) $-\Delta \mathbf{F}$, we find that the transverse and longitudinal fields solve the Helmholtz wave equation

$\triangle \mathbf{E}_{\mathrm{T}}(\mathbf{r}, \omega)+k_{\mathrm{T}}^{2}(\omega) \mathbf{E}_{\mathrm{T}}(\mathbf{r}, \omega)=0$,

$\triangle \mathbf{E}_{\mathrm{L}}(\mathbf{r}, \omega)+k_{\mathrm{L}}^{2}(\omega) \mathbf{E}_{\mathrm{L}}(\mathbf{r}, \omega)=0$,

where the transverse and longitudinal wavenumbers are given by

$k_{\mathrm{T}}^{2}(\omega)=\left(\frac{\omega}{c}\right)^{2} \varepsilon(\omega)$

and

$k_{\mathrm{L}}^{2}(\omega)=\frac{\varepsilon(\omega)}{\xi^{2}(\omega)}$,

respectively. Thus, the nonlocal response leads to a longitudinal wave in the metallic medium. This wave is associated with charge density waves but not with radiation as long as $\mathbf{E}_{\mathrm{L}}$ does not contribute to the magnetic field $\mathbf{H}$; we have $\mathbf{H}=\left(\mathbf{j} / \omega \mu_{0}\right) \nabla \times \mathbf{E}_{\mathrm{T}}$ and $\nabla \cdot \mathbf{H}=0$

The presence of the longitudinal wave requires an additional boundary condition which is considered together with the usual continuity conditions for the tangential components of $\mathbf{E}$ and $\mathbf{H}$. To derive the additional boundary condition we consider a dielectric medium 1 and a metallic medium 2 separated by an interface $S$. At a point $\mathbf{r}$ on $S$, let $\widehat{\mathbf{n}}$ be the normal unit vector pointing into the dielectric medium 1. The displacement field as given by (63) can be written as (we omit the dependence on frequency $\omega$ )

$\mathbf{D}(\mathbf{r})=\mathbf{D}_{\mathrm{b}}(\mathbf{r})-\frac{\mathrm{j}}{\omega} \mathbf{J}(\mathbf{r})$,

where according to (60), the free current density $\mathbf{J}$ reproduces the nonlocal response, while

$\mathbf{D}_{\mathrm{b}}(\mathbf{r})=\varepsilon_{0} \varepsilon_{\mathrm{b}} \mathbf{E}(\mathbf{r})=\varepsilon_{0} \mathbf{E}(\mathbf{r})+\mathbf{P}(\mathbf{r})$, 
is the displacement field due to bound charges, and $\mathbf{P}$ the polarization vector. From Gauss' electric field law $\nabla \cdot \mathbf{D}_{\mathrm{b}}=\rho$, where $\rho$ is the free charge density, a 'pillbox' argument, and Gauss theorem, we have the boundary condition $\widehat{\mathbf{n}} \cdot\left(\mathbf{D}_{\mathrm{b} 1}-\mathbf{D}_{\mathrm{b} 2}\right)=\rho_{\mathrm{s}}$, where

$\rho_{\mathbf{s}}(\mathbf{r})=\lim _{h \rightarrow 0} h[\rho(\mathbf{r}+h \widehat{\mathbf{n}})+\rho(\mathbf{r}-h \widehat{\mathbf{n}})]$

is the free surface charge density. Assuming that thefree surface charge density vanishes at the interface, i.e., $\rho_{\mathrm{s}}=0$, we obtain the continuity of the normal component of the displacement field due to bound charges, $\widehat{\mathbf{n}} \cdot \mathbf{D}_{\mathrm{b} 1}=\widehat{\mathbf{n}} \cdot \mathbf{D}_{\mathrm{b} 2}$, which in turn, gives the additional boundary condition

$\varepsilon_{1} \widehat{\mathbf{n}} \cdot \mathbf{E}_{1}(\mathbf{r})=\varepsilon_{\mathrm{b}} \widehat{\mathbf{n}} \cdot \mathbf{E}_{2}(\mathbf{r})$,

where $\varepsilon_{1}$ is the permittivity of the dielectric medium 1 . If $\varepsilon_{1} \neq$ $\varepsilon_{\mathrm{b}}$, the normal component of the electric field is discontinuous at the interface. This jump in the electric field is due to the surface charge produced by polarization of the bound electrons both in the dielectric and the metal. Defining the bound surface charge density by $\rho_{\mathrm{sb}}=\widehat{\mathbf{n}} \cdot\left(\mathbf{P}_{1}-\mathbf{P}_{2}\right)$, we get from (75) and the continuity of $\widehat{\mathbf{n}}$. $\mathbf{D}_{\mathrm{b}}$,

$\rho_{\mathrm{sb}}=\varepsilon_{0} \widehat{\mathbf{n}} \cdot\left(\mathbf{E}_{2}-\mathbf{E}_{1}\right)$.

On the other hand, from the continuity equation $\nabla \cdot \mathbf{J}=-\mathrm{j} \omega \rho$, we have the boundary condition $\widehat{\mathbf{n}} \cdot\left(\mathbf{J}_{1}-\mathbf{J}_{2}\right)=-\mathrm{j} \omega \rho_{\mathrm{s}}$. Again, under the assumption that the free surface charge density vanishes at the interface, we obtain the continuity of the normal component of the free current density $\widehat{\mathbf{n}} \cdot \mathbf{J}_{1}=\widehat{\mathbf{n}} \cdot \mathbf{J}_{2}$. As dielectrics do not support free currents (there are no free electrons in dielectrics), it follows that $\mathbf{J}_{1}=0$. Hence, at the interface, the normal component of the free current density in the metal vanishes, i.e., $\widehat{\mathbf{n}} \cdot \mathbf{J}_{2}=0$. In other words, no free electrons are moving across the metal surface. From (74) it is then apparent that the continuity of the normal component of the displacement field due to bound charges, and the continuity of the normal component of the free current density imply the continuity of the displacement field, $\widehat{\mathbf{n}} \cdot \mathbf{D}_{1}=\widehat{\mathbf{n}} \cdot \mathbf{D}_{2}$. A few comments are in order on this topic:

1. The boundary condition (77) is equivalent to the assumption that the static electron density $n_{0}$ has a step profile, i.e., $n_{0}$ is constant in metal and rapidly goes to zero at the interface.

2. Taking divergence of the Maxwell equation for the magnetic field corresponding to the vector wave equation (57)

$$
\nabla \times \mathbf{H}(\mathbf{r})=\mathrm{j} \omega \varepsilon_{0} \varepsilon_{\mathrm{b}} \mathbf{E}(\mathbf{r})+\mathbf{J}(\mathbf{r}),
$$

yields

$$
\mathrm{j} \omega \varepsilon_{0} \nabla \cdot\left[\varepsilon_{\mathrm{b}} \mathbf{E}(\mathbf{r})\right]+\nabla \cdot \mathbf{J}(\mathbf{r})=0,
$$

so that by means of a 'pillbox' argument, Gauss theorem, and the continuity of the normal component of the free current density, we rediscover (77).

\section{References}

[1] Maier SA. Plasmonics: Fundamentals and Applications. Springer; 2007.

[2] Pelton M, Bryant G. Introduction to Metal-Nanoparticle Plasmonics. John Wiley \& Sons; 2013.

[3] Le Ru EC, Etchegoin PG. Single-molecule surface-enhanced raman spectroscopy. Annu Rev Phys Chem 2012;63:65-87.

[4] Wubs M, Mortensen A. Nonlocal Response in Plasmonic Nanostructures. In: Bozhevolnyi SI, Martin-Moreno L, Garcia-Vidal F, editors. Quantum Plasmonics. Switzerland: Springer; 2017. p. 279-302.

[5] David C, García de Abajo FJ. Spatial nonlocality in the optical response of metal nanoparticles. J Phys Chem C 2011;115:19470-5.

[6] Esteban R, Zugarramurdi A, Zhang P, Nordlander P, García-Vidal FJ, Borisov AG, Aizpurua J. A classical treatment of optical tunneling in plasmonic gaps: extending the quantum corrected model to practical situations. Faraday Discuss 2015; 178:151-83.

[7] Zhu W, Esteban R, Borisov AG, Baumberg JJ, et al. Quantum mechanical effects in plasmonic structures with subnanometre gaps. Nature Commun 2016;7:11495.
[8] Zuloaga J, Prodan E, Nordlander P. Quantum description of the plasmon resonances of a nanoparticle dimer. Nano Lett 2009;9:887-91.

[9] Teperik TV, Nordlander P, Aizpurua J, Borisov AG. Quantum effects and nonlocality in strongly coupled plasmonic nanowire dimers. Opt Express 2013;21:27306-25.

[10] Mortensen NA. Nonlocal formalism for nanoplasmonics: phenomenological and semi-classical considerations. Photonics Nanostruct 2013;11:303-16.

[11] Mortensen NA, Raza S, Wubs M, Sø ndergaard T, Bozhevolnyi SI. A generalized non-local optical response theory for plasmonic nanostructures. Nature Commun 2014:5:3809-15.

[12] Raza S, Bozhevolnyi SI, Wubs M, Mortensen NA. Nonlocal optical response in metallic nanostructures. Topical Rev J Phys Condens Matter 2015;27:3204-300.

[13] Toscano G, Straubel J, Kwiatkowski A, Rockstuhl C, Evers F, Xu H, Mortensen NA. Wubs M. Resonance shifts and spill-out effects in self-consistent hydrodynamic nanoplasmonics. Nat Commun 2015;6:7132.

[14] McMahon JF, Gray SK, George C, Schatz GC. Calculating nonlocal optical properties of structures with arbitrary shape. Phys Rev B 2010;82:035423.

[15] Luo Y, Fernandez-Dominguez AI, Wiener A, Maier SA, Pendry JB. Surface plasmons and nonlocality: a simple model. Phys Rev Let 2013;111:093901.

[16] Esteban R, Borisov AG, Nordlander P, Aizpurua J. Bridging quantum and classical plasmonics with a quantum-corrected model. Nat Commun 2012;3:825.

[17] David C, García de Abajo FJ. Surface plasmon dependence on the electron density profile at metal surfaces. ACS Nano 2014;8:9558-66.

[18] Yan W, Wubs M, Mortensen NA. Projected dipole model for quantum plasmonics. Phys Rev Lett 2015;115:137403.

[19] Monreal RC, Antosiewicz TJ, Apell SP. Competition between surface screening and size quantization for surface plasmons in nanoparticles. N J Phys 2013;15:083044.

[20] Toscano G, Raza S, Jauho A, Mortensen NA, Wubs M. Modified field enhancement and extinction by plasmonic nanowire dimers due to nonlocal response. Opt Express 2012;20:4176-88.

[21] Ch T, Yan W, Hsieh W, Sun G, et al. On the origin of nonlocal damping in plasmonic monomers and dimers. Int J Mod Phys B 2017;31:17400005.

[22] Kahnert M. Numerical solutions of the macroscopic maxwell equations for scattering by non-spherical particles: a tutorial review. J Quant Spectrosc Radiat Transfer 2016;178:22-37.

[23] Gallinet B, Butet J, OJF M. Numerical methods for nanophotonics: standard problems and future challenges (review). Laser Photonics Rev 2015;9(6):577-603.

[24] Yurkin MA. Computational Approaches for Plasmonics. In: Della Sala F, D’Agostino S, editors. Handbook of Molecular Plasmonics. Publisher: Pan Stanford Publishing; 2013. p. 83-135. Chapter: 2

[25] Tafove A, Hagness S. Computational Electrodynamics: The Finite-Difference Time Domain Method. Third edition. Boston, MA: Artech House; 2005.

[26] Jin JM. The Finite Element Method in Electromagnetics. Third edition. Wiley-IEEE Press; 2014

[27] Khlebtsov NG. T-matrix method in plasmonics: an overview. J Quant Spectrosc Radiat Transfer 2013;123:184-217.

[28] Ch H, Smajic J, Agio M. Numerical Methods for the Electrodynamic Analysis of Nanostructures, in Nanoclusters and Nanostructured Surfaces. Valencia, California, USA: American Scientifc Publishers; 2010. p. 207-74.

[29] Eremin YA, Sveshnikov AG. Mathematical models in nanooptics and biophotonics problems on the base of discrete sources method. Comput Math Math Phys 2007;47(2):262-79.

[30] YuA E, Wriedt T, Hergert W. Analysis of the scattering properties of 3d nonspherical plasmonic nanoparticles accounting for nonlocal effects. J Mod Opt 2018. doi:10.1080/09500340.2018.1459911.

[31] Yu E, Wriedt T. Analysis of scattered field enhancement in the evanescent wave area based on the discrete sources method. J Quant Spectrosc Radiat Transfer 2014;146:235-43.

[32] Johnson PB, Christy RW. Optical constants of the noble metals. Phys Rev B 1972;6:4370-9.

[33] YuA E, Grishina NV, Eremina E, Wriedt T. Analysis of plasmonic resonances of two paired noble metal spheroids via the discrete sources method. J Mod Opt 2013;60(7):529-37.

[34] Nordlander P, Oubre C, Prodan E, Li K, Stockman MI. Plasmon hybridization in nanoparticle dimers. Nano Lett 2004;4(5):899-908.

[35] Yan W, Wubs M, Mortensen NA. Hyperbolic metamaterials: non local response regularizes broadband supersingularity. Phys Rev B 2012;86:205429.

[36] Mochán WL, del Castillo MM, Barrera RG. Effect of plasma waves on the optical properties of metal-insulator superlattices. Phys Rev B 1987;35:1088-98.

[37] Ciracì C, Hill RT, Mock JJ, Urzhumov Y, et al. Probing the ultimate limits of plasmonic enhancement. Science 2012;337(6098):1072-4.

[38] Cacciola A, Iatì MA, Saija R, Borghese F, et al. Spectral shift between the nearfield and far-field optoplasmonic response in gold nanospheres, nanoshells, homo- and hetero-dimers. J Quant Spectrosc Radiat Transfer 2017;196:97-106.

[39] Doicu A, Yu E, Wriedt T. Acoustic and Electromagnetic Scattering Analysis Using Discrete Sources. San Diego: Academic Press; 2000.

[40] Filter R, Bösel C, Toscano G, Lederer F, Rockstuhl C. Nonlocal effects: relevance for the spontaneous emission rates of quantum emitters coupled to plasmonic structures. Opt Lett 2014;39:6118. 